\title{
Optic Cup Segmentation using U-Net Architecture on Retinal Fundus Image
}

\author{
Pulung Hendro Prastyo, Amin Siddiq Sumi, Annis Nuraini \\ Department of Electrical and Information Technology Engineering, Universitas Gadjah Mada, Yogyakarta, Indonesia
}

\section{ARTICLE INFORMATION}

Received: August $10^{\text {th }}, 2020$

Revised: September $21^{\text {st }}, 2020$

Available online: September $30^{\text {th }}, 2020$

\section{KEYWORDS}

Computer Vision, Deep Learning, Image

Segmentation, Optic Cup Segmentation, U-Net

CORRESPONDENCE

E-mail: pulung.hendro@mail.ugm.ac.id

\section{A B S T T R A C T}

Retinal fundus images are used by ophthalmologists to diagnose eye disease, such as glaucoma disease. The diagnosis of glaucoma is done by measuring changes in the cup-to-disc ratio. Segmenting the optic cup helps petrify ophthalmologists calculate the CDR of the retinal fundus image. This study proposed a deep learning approach using U-Net architecture to carry out segmentation task. This proposed method was evaluated on 650 color retinal fundus image. Then, U-Net was configured using 160 epochs, image input size $=128 \times 128$, Batch size $=32$, optimizer $=$ Adam, and loss function $=$ Binary Cross Entropy. We employed the Dice Coefficient as the evaluator. Besides, the segmentation results were compared to the ground truth images. According to the experimental results, the performance of optic cup segmentation achieved $98.42 \%$ for the Dice coefficient and loss of 0.15 . These results implied that our proposed method succeeded in segmenting the optic cup on color retinal fundus images.

\section{INTRODUCTION}

A retinal fundus image is an essential tool for documenting the optic nerve's health, vitreous, macula, retina, and blood vessels [1]. Ophthalmologists used a fundus camera to take the retinal image. They use the retinal image to diagnose eye disease, such as glaucoma. Glaucoma is a significant cause of global blindness that cannot be cured [2]-[5]. Glaucoma disease can change the cup region's shape, which is the center portion of the optic nerve head $(\mathrm{ONH})[6]$. The changes can be used as a parameter for the early indicator of glaucoma. The $\mathrm{ONH}$ transmits visual information from retina to the brain.

There are no initial glaucoma symptoms but will gradually damage the optic nerve and then lead to blindness. Thus, it is crucial to detect glaucoma as early as possible so that it can prevent visual damage [3][4]. Physiologically, glaucoma is indicated by increased optic cup excavation. The increasing size of the optic cup will impact the size of the optic disc, this relation known as a cup-to-disc ratio (CDR) [4][7]. It means ophthalmologists can diagnose glaucoma progression using the value of CDR measurement. The optic cup and optic disc segmentation will support to calculate the CDR from the retinal image.

According to Figure 1, the optic cup is a bright central zone [3] [5][8]. The intensity of color pixel and associated gradients form the basis of a method for cup segmentation. In this research work, we proposed a Deep Learning approach using U-Net architecture to segment the optic cup.

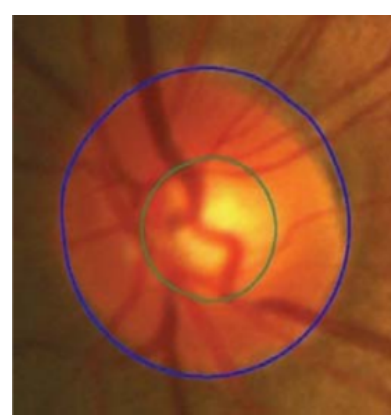

(a) Normal

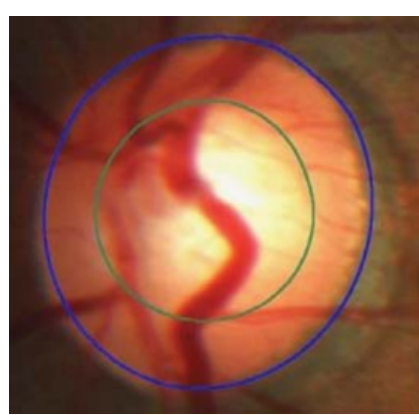

(b) Glaucoma
Figure. 1. Optic Nerve Head Structure. The Optic Disk (Blue) and The Optic Cup (Green)

\section{RELATED WORK}

Several approaches have been developed for optic cup segmentation on retinal fundus images. Almazroa et al. [6] proposed an automatic system for optic cup segmentation based on the image intensity and vessel kinks. Vessel extraction was done using top-hat transform. While detecting the curvature of the blood vessels, the authors used Otsu's function. The curvature indicated the cup boundary. Formerly, the Fuzzy Interval Type-II entropy was implemented to the cup thresholding. Thus, the Hough transform was applied to the cup boundaries. The accuracy of cup detection was $72.5 \%$.

Attribution-NonCommercial 4.0 International. Some rights reserved 
In 2017, Nugroho et al. [4] segmented optic cup to calculate the vertical cup-to-disc ratio. The authors used the mix of morphological reconstruction and convex hull for optic cup segmentation. Three main steps were conducted, i.e., preprocessing, vessel removal, and segmentation. The accuracy level at $99.13 \%$, sensitivity level at $80.68 \%$, and a specificity level at $99.53 \%$. The performance indicated that the proposed method was succeeded.

In the last few years, a deep learning approach has been used extensively to classify, segment, and detect medical images. Alom et al. [9] employed Recurrent Convolutional Neural Network (RCNN) based on U-Net architecture to segment medical image. The authors select U-Net architecture because there are several advantages, such as better feature representation for segmentation, and easy to design a U-Net architecture for medical image segmentation. The proposed methods were applied to segment blood vessels in retina images, skin cancer, and lung lesion. The result showed better performance in both the training and testing phase.

Different from Alom et al., Qin et al. [5] proposed modification of fully convolutional network (FCN) and the Inception building blocks in GoogleNet to segment optic cup. The authors used the Hough Circle Transform algorithm to locate the optic disc then cut the optic disc automatically. In the pre-processing steps, the authors removed the red channel in the retinal image because it contains less useful information. After that, the result was applied to modify a fully convolutional network and Inception structure in GoogleNet to perform optic cup segmentation. The experimental result showed that the method could effectively segment the optic cup compared to current state-of-the-art methods.

The use of U-Net architecture for optical cup segmentation was done by Joshua et al. [10]. The authors improved U-Net Convolutional Neural Network to segment optic cup from retinal image. The U-Net architecture is modified so that the number of parameters used is less compared to U-Net in general. The result showed that the proposed architecture outperformed existing techniques on the Dice score metric and prediction time.

Besides being used for optical cup segmentation, the U- Net model is also employed for segmenting the fundus retinal vessels. This study was conducted by Pan et al. [11]. The U-Net model was used to solve several problems experienced by existing methods for retinal vascular segmentation, such as low microvascular segmentation and incorrect pathological information segmentation. The experimental results showed the segmentation accuracy was $96.50 \%$, the sensitivity was $93.1 \%$, and the specificity was $98.63 \%$.

Based on previous studies, U-Net obtained a good performance. Therefore, in this study, we employed U-Net which its architectures and variables are modified using ORIGA dataset about glaucoma disease to segment Optic Cup better.

\section{METHOD}

In this research work, we used a deep learning algorithm based on U-Net architecture for optic cup segmentation. We employed
U-Net architecture because U-Net architecture showed an excellent result of a medical image segmentation based on the previous study. The work scheme of our proposed method is shown in Figure 2.

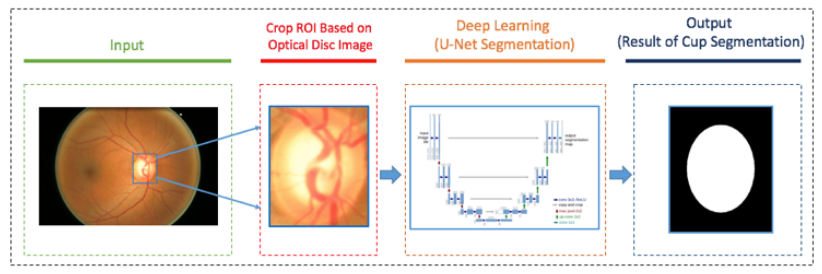

Figure. 2. Work Scheme of Proposed Method

\section{Dataset}

We used the ORIGA dataset in our experiment [1]. The dataset consists of 650 images of the color retinal fundus with the extension (.jpg) and ground truth with the extension (.mat). An example of a color retinal fundus image can be seen in Figure. 3 (a) and ground truth can be seen in Fig. 3 (b). The retinal images were collected by the Singapore Malay Eye Study (SiMES). The purpose of SiMES is to determine the causes and risk factors of blindness and visual damage in the Singapore Malay community. The SiMES tested 3,280 Malay adults from ages 40 to 80 years, and 149 were glaucoma patients.

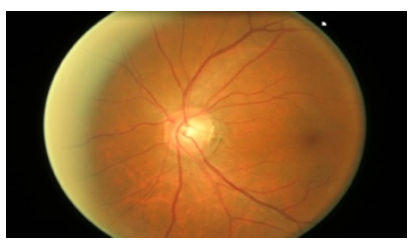

(a) An Image of Color Retinal Fundus

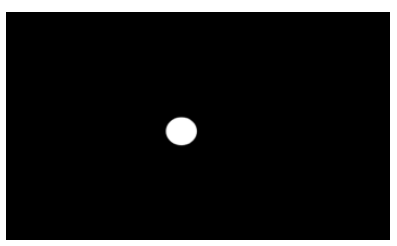

(b) A Ground Truth
Figure. 3. Retinal Fundus Image

\section{Pre-processing}

Before segmenting the image, the ground truth (mask) image was changed to (.png) so that an algorithm could process it. To get the Optic Disk (OD) mask, we used the equation (disc $=$ double (mask $>0)$ ), while for Optic Cup (OC), we employed the equation $($ cup $=$ double $($ mask $>1))$.

After that, we took the Region of Interest (ROI) from the retinal fundus image by using the ground truth from OD to take OC's closest area. We employed the boundingRect function provided by the OpenCV-python library to obtain ROI from OC images. The method can be seen in Figure. 4.

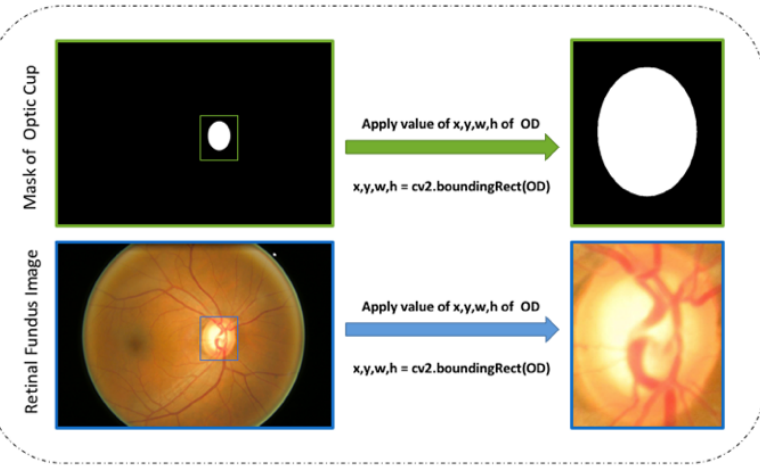

Figure 4. Work Scheme of How to Take ROI 
In Figure 4, the boundingRect function took the values $\mathrm{x}, \mathrm{y}$, weight, and height in an OD image. After that, the value was implemented into the ground truth and retinal fundus images.

\section{Proposed Method}

After pre-processing, 650 image data were divided into 448 image data as $70 \%$ training data, 192 image data as $30 \%$ validation data, and ten image data as testing data.

In this study, U-Net's Deep Learning Algorithm was employed to segment OC Image and Google Collab was used to run U-Net. UNet architecture is illustrated in Figure 5. It consists of three parts those are encoding, bridge, and decoding [12]. The encoding part/contraction path (left side) modifies the input image into condense representation following the typical architecture of a convolutional network [13]. It consists of two $3 \times 3$ convolutions and a $2 \times 2$ max pooling operation, and each convolution is pursued by a rectified linear unit (ReLU). The decoding process restores the representation to pixel-wise outputs of the same size from the input image by the up-sampling operation. The role of the bridge is to tie between encoding and decoding parts. Whereas in the decoding part/expansive path (right side) consists of an upsampling of feature map pursued by a $2 \times 2$ convolution, which halves the number of feature channels combined with feature maps cutting from the contract line, and two $3 \times 3$ convolutions, each pursued by ReLU. Cropping is needed because of the loss of border pixels at each convolution. In the last layer, $1 \mathrm{x} 1$ convolution is used to map each 64-component feature vector to the desired number of classes.

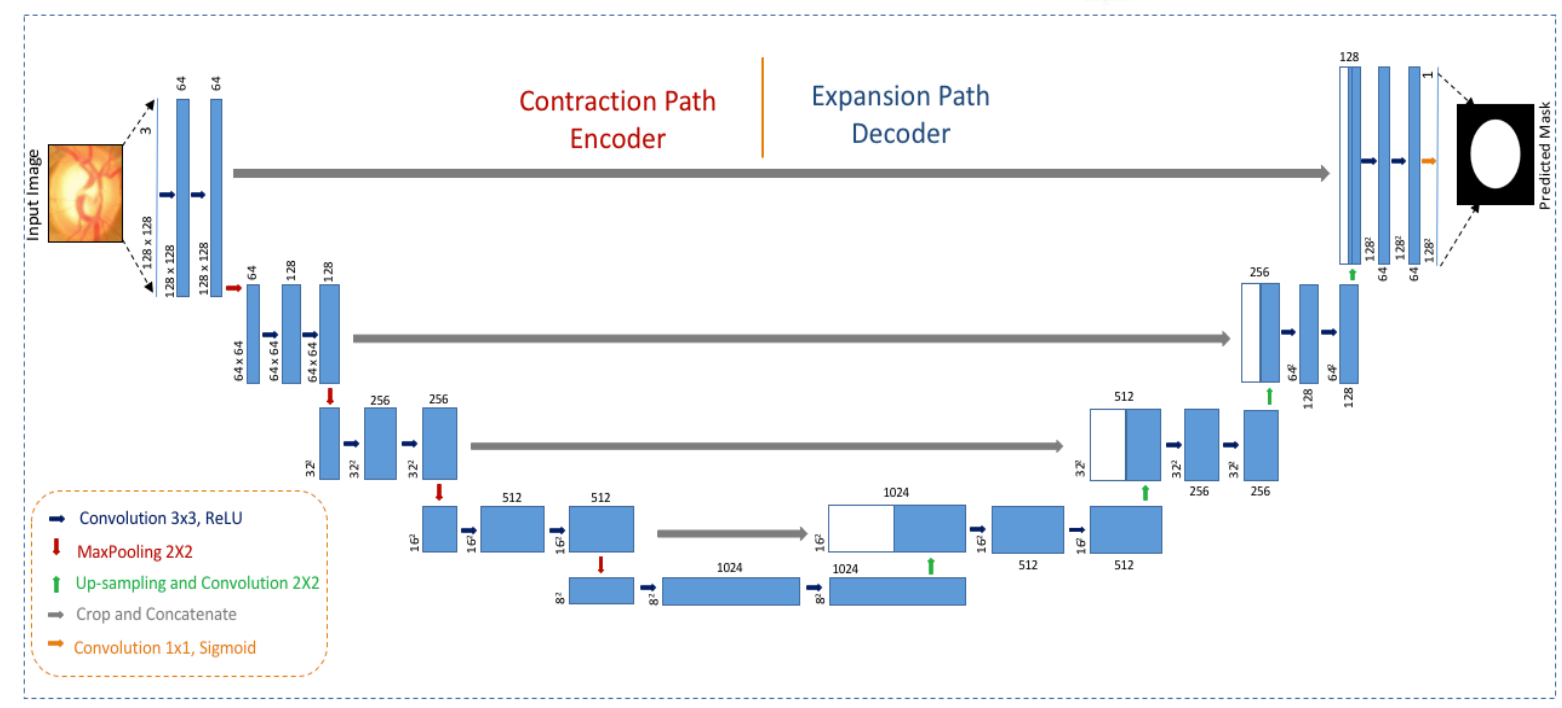

Figure. 5. The Architecture of Modified U-Net Algorithm

The configuration of the U-Net algorithm is described in Table 1 . We used the size of the input image (128x128), batch size (32), and filter $(64,128,256,512,1024)$. For the training optimizer, we employed Adam (Adaptive learning rate optimization algorithm). Adam combines RMSProp and Stochastic Gradient Descent (SGD) method; thus, Adam has a good learning level.

Table 1. Configuration of U-Net

\begin{tabular}{ll}
\hline Variable & Configuration \\
\hline Epoch & 160 \\
Size of Input Image & $128 \times 128$ \\
Batch Size & 32 \\
Filter & $64,128,256,512,1024$ \\
Optimizer & Adam \\
Loss Function & Binary Cross Entropy \\
Matric & Dice Coefficient \\
\hline
\end{tabular}

Besides, we used binary cross-entropy for loss function because we applied sigmoid with range 0 to 1 . It indicates probability distribution so that we assume our task is a classification task. Finally, we used dice coefficient metrics to evaluate the result of U-Net segmentation and ground truth. The higher the dice coefficient value, the better the accuracy. The equation of the dice coefficient is two times the area of overlap divided by the total number of pixels on both images.

\section{RESULTS AND DISCUSSION}

According to the experimental results, our proposed method achieved a dice coefficient score of $98.42 \%$ and a loss of 0.15 using 160 epochs. The selection of epochs is based on the performance of the algorithm in Figure 6 which starts to stabilize at 100-160 epochs. For validation, U-Net obtained a dice coefficient score of $92.68 \%$ and a loss of 0.28 with a computational time of 3 hours 14 minutes 3 seconds. Based on the results, our proposed method can be considered as a robust method for optic cup segmentation. The graph of evaluation results using the dice coefficient can be seen in Figure 6. 


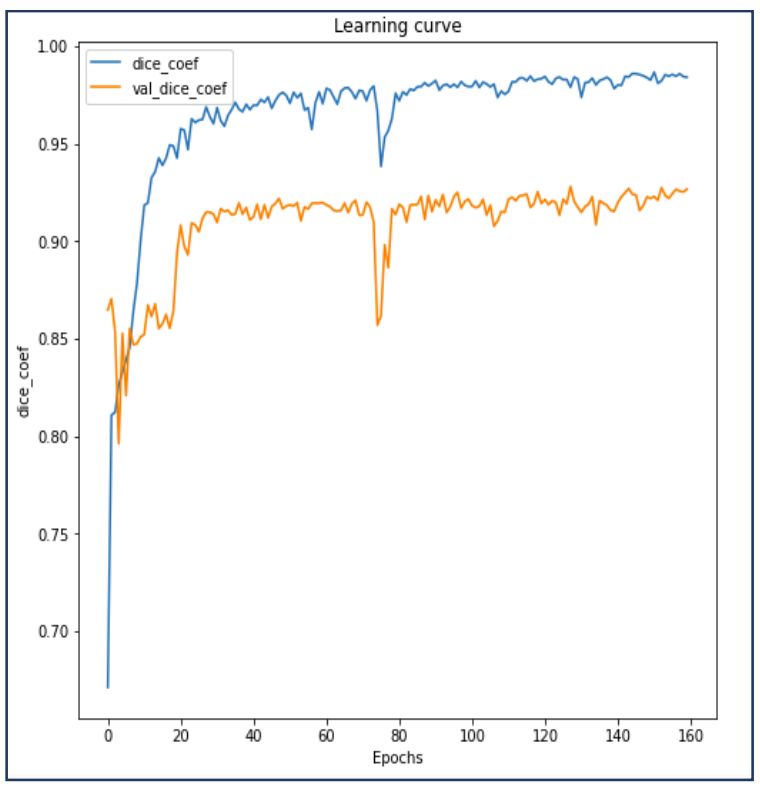

Figure. 6. The Graph of Evaluation Results

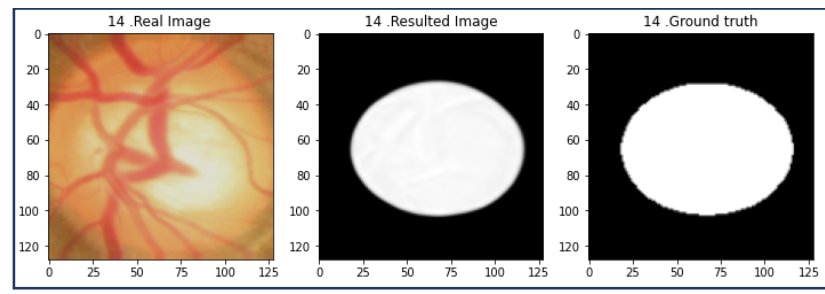

(a)

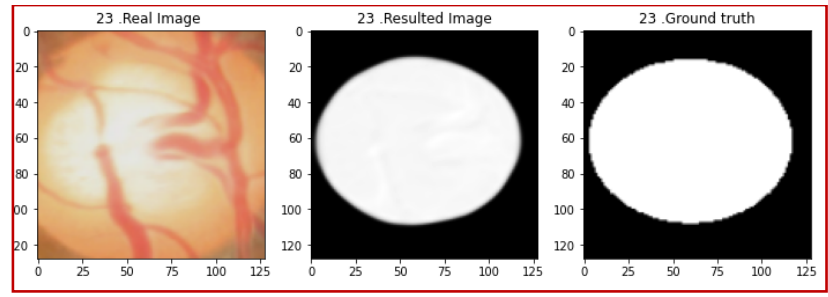

(b)

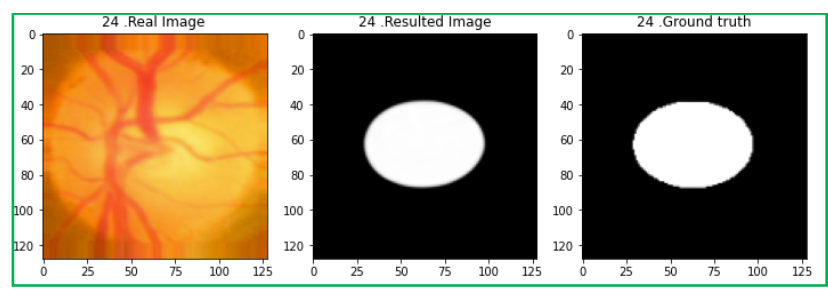

(c)

Figure. 7. Comparison of U-Net Result with Ground Truth

The results of OC image segmentation can be seen in Figure 7. The image results of segmentation using U-Net were compared to ground truth images. The results of U-Net segmentation were exact with the ground truth image, which was suitable with the high dice coefficient score obtained by U- Net. Consequently, the resulted images look more similar to raw data than ground truth because the ground truth pictures do not show any arches.

The results were not much different from the ground truth image for testing data in which these images were not included in the training process. It indicates that our proposed method is robust and competitive for segmenting the Optic Cup (OD) on the color retinal fundus image. The images can be seen in Figure 8.

108 Pulung Hendro Prastyo

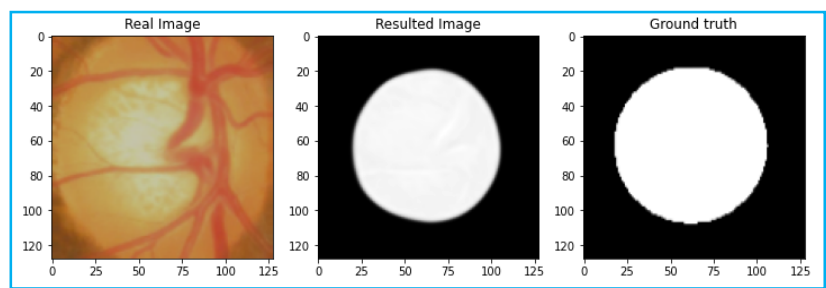

(a)

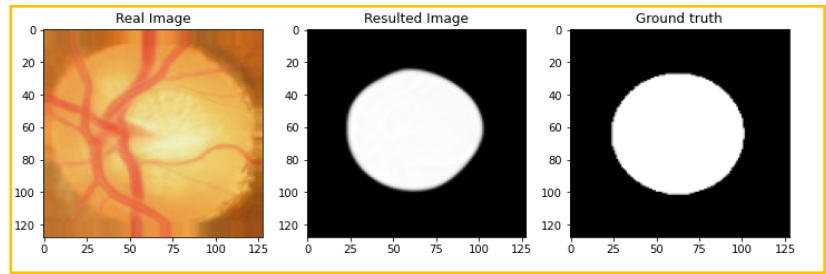

(b)

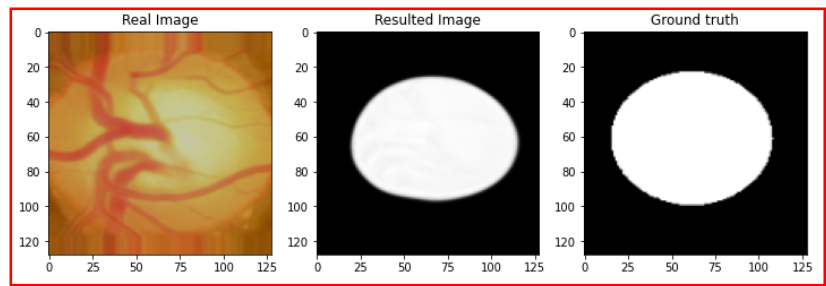

(c)

Figure. 8. Comparison of U-Net result with ground truth using testing data

\section{CONCLUSIONS}

In this study, we proposed a deep learning approach using U-Net architecture to carry out the segmentation task. Our proposed method is evaluated on 650 color retinal fundus images. Then, UNet is configured using 160 epochs, image input size $=128 \times 128$, Batch size $=32$, optimizer $=$ Adam, and loss function $=$ Binary Cross Entropy. We employ the Dice Coefficient as the evaluator. Moreover, the segmentation results are compared to the ground truth image. According to the experimental results, the performance of optic cup segmentation has achieved $98.42 \%$ for the Dice coefficient and loss of 0.15 . The results imply that our proposed method succeeds in segmenting the optic cup on color retinal fundus images very well.

As for our method's weaknesses, we have not been able to detect and crop ROI Optic Cup (OC) automatically. We still use the Optic Disc (OD) size as the ROI OC. For further research, researchers can use the localization method using green or red channels to detect the ROI of the Optic Cup. For the U-Net Algorithm, it can be improved by configuring larger input sizes, batch sizes, and more epochs so that segmentation can be more precise.

\section{REFERENCES}

[1] Z. Zhang et al., "ORIGA-light: An online retinal fundus image database for glaucoma analysis and research," in 2010 Annual International Conference of the IEEE Engineering in Medicine and Biology, 2010, pp. 30653068, doi: 10.1109/IEMBS.2010.5626137.

[2] Y. Jiang, N. Tan, and T. Peng, "Optic Disc and Cup Segmentation Based on Deep Convolutional Generative 
Adversarial Networks," IEEE Access, vol. 7, pp. 6448364493, 2019, doi: 10.1109/ACCESS.2019.2917508.

[3] G. D. Joshi, J. Sivaswamy, and S. R. Krishnadas, "Optic disk and cup segmentation from monocular color retinal images for glaucoma assessment," IEEE Trans. Med. Imaging, vol. 30, no. 6, pp. 1192-1205, 2011, doi: 10.1109/TMI.2011.2106509.

[4] H. A. Nugroho, W. K. . Oktoeberza, A. Erasari, A. Utami, and C. Cahyono, "Segmentation of Optic Disc and Optic Cup in Colour Fundus Images Based on Morphological Reconstruction," in 9th International Conference on Information Technology and Electrical Engineering (ICITEE), 2017, $\quad$ pp. 1-5, doi: 10.1017/CBO9781107415324.004.

[5] P. Qin, L. Wang, and H. Lv, "Optic disc and cup segmentation based on deep learning," in 2019 IEEE 3rd Information Technology, Networking, Electronic and Automation Control Conference, ITNEC 2019, 2019, pp. 1835-1840, doi: 10.1109/ITNEC.2019.8729455.

[6] A. Almazroa, S. Alodhayb, R. Burman, W. Sun, K. Raahemifar, and V. Lakshminarayanan, "Optic cup segmentation based on extracting blood vessel kinks and cup thresholding using Type-II fuzzy approach," in 2nd International Conference on Opto-Electronics and Applied Optics: Advances in Optical Sciences and Engineering II, IEM OPTRONIX 2015, 2015, pp. 2-6, doi: 10.1109/OPTRONIX.2015.7345519.

[7] D. W. K. Wong, J. Liu, J. H. Lim, H. Li, and T. Y. Wong, "Automated detection of kinks from blood vessels for optic cup segmentation in retinal images," in Medical Imaging 2009: Computer-Aided Diagnosis, 2009, vol. 7260, pp. 459-466, doi: 10.1117/12.810784.

[8] R. Ingle and P. Mishra, "Cup Segmentation by Gradient Method for the Assessment of Glaucoma from Retinal Image," Int. J. Eng. Trends Technol., vol. 4, no. 6, pp. 2540-2543, 2013.

[9] M. Z. Alom, M. Hasan, C. Yakopcic, T. M. Taha, and V. K. Asari, "Recurrent Residual Convolutional Neural Network based on U-Net (R2U-Net) for Medical Image Segmentation," CoRR, vol. abs/1802.06955, 2018, [Online]. Available: http://arxiv.org/abs/1802.06955.

[10] A. O. Joshua, F. V. Nelwamondo, and G. Mabuza-Hocquet, "Segmentation of Optic Cup and Disc for Diagnosis of Glaucoma on Retinal Fundus Images," in Proceedings 2019 Southern African Universities Power Engineering Conference/Robotics and Mechatronics/Pattern Recognition Association of South Africa, SAUPEC/RobMech/PRASA 2019, 2019, pp. 183-187, doi: 10.1109/RoboMech.2019.8704727.

[11] P. Xiuqin, Q. Zhang, H. Zhang, and S. Li, “A fundus retinal vessels segmentation scheme based on the improved deep learning u-net model," IEEE Access, vol. 7, pp. 122634
122643, 2019, doi: 10.1109/ACCESS.2019.2935138.

[12] W. Zhang, P. Tang, L. Zhao, and Q. Huang, "A comparative study of U-nets with various convolution components for building extraction," in 2019 Joint Urban Remote Sensing Event, JURSE 2019, 2019, pp. 1-4, doi: 10.1109/JURSE.2019.8809055.

[13] O. Ronneberger, P. Fischer, and T. Brox, "U-Net: Convolutional Networks for Biomedical Image Segmentation," in Medical Image Computing and Computer-Assisted Intervention -- MICCAI 2015, 2015, pp. 234-241. 